Pacific Journal of Mathematics

UNIVERSAL APPROXIMATION BY REGULAR WEIGHTED 


\title{
UNIVERSAL APPROXIMATION BY REGULAR WEIGHTED MEANS
}

\author{
B. ThORPE AND L. TOMM
}

In this paper we shall improve upon the results by K. Faulstich, W. Luh and L. Tomm by (i) considering power series representing other meromorphic functions $f$, (ii) using a regular weighted means method $D$ to obtain the overconvergence property and (iii) showing that $D$ has a universal property with respect to analytic continuation i.e. for every simply connected region $G$ which contains the open disc of convergence of the Maclaurin series of $f$ but no pole of $f$, there is a subsequence of the $D$-transform of the $n$th partial sums of the Maclaurin series of $f$ that converges to $f$ uniformly on compact subsets of $G$.

1. Introduction. The behaviour of partial sums of power series outside their circle of convergence has been studied by various authors c.f. [4], [9]. Power series that have subsequences of partial sums which converge at points outside their circle of convergence are said to be overconvergent. In [1], Chui and Parnes proved the existence of a power series $\Pi$, convergent in the open unit disc and with the following universal property with respect to overconvergence:

Given any compact set $L$ which does not separate the plane and does not intersect the closed unit disc $\Delta$, and given any function $g$ that is continuous on $L$ and holomorphic in the interior of $L$ (i.e. $g \in A(L)$ ), there exists $a$ subsequence of the partial sums of $\Pi$ that converges to $g$ uniformly on $L$.

More recently, it was shown in [12], that a power series exists that is absolutely convergent in $\Delta$ and overconverges almost everywhere outside $\Delta$ to any given measurable function $f$. Moreover such power series are dense in the Banach space $A(\Delta)$ (with the uniform norm). On the other hand, not all power series are overconvergent. For instance, the geometric series has the property that no subsequence of its partial sums converges at any point outside $\Delta$. However, even in this case, if we consider a summability transform of its sequence of $n$th partial sums (as in [5], [7]) then it is possible to obtain overconvergence properties. More precisely, in [6] Luh proved the existence of a summability method $A$ such that the $A$-transform of the $n$th partial sums of the geometric series $\left(\psi_{n}^{A}\right)_{n \geq 0}$ converges on the interior of $\Delta$ to $1 /(1-z)$ and has the following universal overconvergence property: 
Given any compact set $L$ which does not separate the plane and does not intersect $\Delta$, and given any function $g \in A(L)$, there exists a subsequence $\left(\psi_{k_{n}}^{A}\right)$ of $\left(\psi_{n}^{A}\right)$ that converges to $g$ uniformly on $L$.

Using a summability approximation theorem obtained in [10], it was shown in [3] that the method $A$ constructed by Luh could be chosen to be regular and in fact it could be a regular 'generalised weighted means method' or 'generalised Riesz method' previously introduced by Faultstich (see [2]). Furthermore, as a consequence of the results in [3], the sequence $\left(\psi_{n}^{A}\right)$ provides an analytic continuation of the geometric series into a fixed simply connected region $G$ containing the open unit disc but not the point 1.

Notation. For every set $S \subset \mathbf{C}$, let $S$ denote the interior, $\bar{S}$ the closure and $S^{c}$ the complement of $S$. A sequence of functions $\left(f_{n}\right)$ will be called compactly convergent to a function $f$ on $S$ if it converges to $f$ uniformly on every compact subset of $S$. We use the following abbreviations throughout: $\Delta_{r}=\{z \in \mathbf{C}:|z| \leq r\}$ for $r \geq 0, \Delta=\Delta_{1}, \mathbf{N}_{0}$ for the set of non-negative integers and $\mathbf{C}_{\infty}=\mathbf{C} \cup\{\infty\}$. If $K$ is a compact subset of $\mathrm{C}$ then $A(K)$ denotes the Banach space of all functions that are continuous on $K$ and holomorphic on $\stackrel{\circ}{K}$.

A matrix $D=\left(d_{n, k}\right)$ defining a sequence to sequence summability method is called a weighted means method if there is a sequence $\left(d_{k}\right)_{k \geq 0}$ such that $D_{n}=d_{0}+d_{1}+\cdots+d_{n} \neq 0$ for $n=0,1,2, \ldots$ and

$$
d_{n, k}= \begin{cases}d_{k} / D_{n} & \text { if } 0 \leq k \leq n, \\ 0 & \text { if } k>n .\end{cases}
$$

Cf. [9] where the notation $\mathscr{M}(d)$ is used and [4] where $(\bar{N}, d)$ is used.

It is well known that $D$ is regular (i.e. finite limit preserving) if and only if the following two conditions hold

$$
\begin{gathered}
\lim _{n \rightarrow \infty} D_{n}=\infty, \\
\sum_{k=0}^{n}\left|d_{k}\right|=O\left(\left|D_{n}\right|\right) .
\end{gathered}
$$

2. Statement of the results. First we describe the class of functions to which our results apply. Throughout this paper let $f$ denote a function that is meromorphic in $\mathbf{C}$ and has a Maclaurin series expansion

$$
f(z)=\sum_{m=0}^{\infty} c_{m} z^{m}
$$


whose radius of convergence, $r$, is positive. Furthermore we also require that the coefficients $c_{m}$ satisfy the condition

$$
\lim _{m \rightarrow \infty} R^{m} c_{m}=\infty \quad \text { for every } R>r .
$$

It was proved in [11] that (C) holds if

$f$ has exactly one pole on the circle $|z|=r$, or if

$$
\lim _{m \rightarrow \infty} R^{m} \tilde{c}_{m}=\infty \quad \text { for every } R>r
$$

where $\tilde{f}(z)=\sum_{m=0}^{\infty} \tilde{c}_{m} z^{m}$ is any rational function which has the same poles and the same singular parts as $f$ on $\Delta_{r}$. It is clear that not every meromorphic function satisfies (C) (for instance, if $f$ is even then it does not satisfy $(\mathrm{C})$ ).

We use $P$ to denote the set of poles of $f$.

If $A=\left(a_{n, k}\right)$ is a row finite summability method then $\left(\sigma_{n}^{A}(z)\right)$ will denote the sequence of $A$-transforms of the $n$th partial sums of the series in (4), that is, for all $z \in \mathbf{C}$ and $n=0,1, \ldots$

$$
\sigma_{n}^{A}(z)=\sum_{k=0}^{\infty} a_{n, k}\left(\sum_{m=0}^{k} c_{m} z^{m}\right) .
$$

We now establish the existence of 'a regular universal weighted means method' $D$ for the function $f$.

THEOREM. There exists a regular weighted means method $D$ with the following property:

For every triple $(G, L, g)$, where

(i) $G$ is any simply connected region that contains $\AA_{r}$ but no pole of $f$,

(ii) $L$ is any compact set which does not separate the plane and contains no point of $P \cup G \cup \Delta_{r}$,

(iii) $g$ is any function in $A(L)$, there exists a subsequence $\left(\sigma_{k_{n}}^{D}\right)$ of $\left(\sigma_{n}^{D}\right)$ such that

$$
\lim _{n \rightarrow \infty} \sigma_{k_{n}}^{D}(z)= \begin{cases}f(z) & \text { compactly on } G, \\ g(z) & \text { uniformly on } L .\end{cases}
$$

REMARK. The weighted means $\left(\sigma_{n}^{D}\right)$ converge to $f$ compactly on $\check{\Delta}_{r}$. This follows directly from the fact that $D$ is regular (see [6]).

Before proving the theorem we draw two corollaries that give further properties of $D$ (cf. Theorems 4 and 5 of [11]).

COROLlARY 1. There exists a subsequence of $\left(\sigma_{n}^{D}\right)$ which converges to $f$ on all of $\mathbf{C} \backslash P$. 
Proof. Let $E=\{\lambda z \mid z \in P, \lambda \geq 1\}$ and $G=\mathbf{C} \backslash E$, the Mittag-Leffler star of $f$. The set $E \backslash P$ consists of countably many open line segments. Let $K_{n}$ be the set of all points in $G$ whose moduli do not exceed $n+1$ and whose distance to the complement of $G$ is at least $1 /(n+1)$ and let $L_{n}$ be the set of all points in $E \backslash P$ whose moduli do not exceed $n+1$ and whose distance to $P$ is at least $1 /(n+1)$.

In view of the theorem we can find for every $n \geq 0$ a subsequence of $\left(\sigma_{m}^{D}\right.$ ) tending to $f$ uniformly on $K_{n} \cup L_{n}$. By choosing a suitable diagonal sequence we obtain a subsequence $\left(\sigma_{m_{n}}^{D}\right)$ such that

$$
\left|\sigma_{m_{n}}^{D}(z)-f(z)\right|<1 /(n+1) \text { for all } z \in K_{n} \cup L_{n} .
$$

Since every point of $\mathbf{C} \backslash P$ belongs to $K_{n} \cup L_{n}$ for large $n$ the sequence $\left(\sigma_{m_{n}}\right)$ does the job.

COROLlaRY 2. The method $D$ of the theorem has the following property: If (i) $\bigsqcup_{r} \subset G_{0}$ and $G_{0}, G_{1}, \ldots$ is a finite or infinite sequence of disjoint simply connected regions that do not contain a pole of $f$, (ii) $f_{0}=f$ and, for each $\nu \geq 1, f_{\nu}$ is holomorphic on $G_{\nu}$, then there exists a subsequence $\left(\sigma_{p_{n}}^{D}\right)$ of $\left(\sigma_{n}^{D}\right)$ such that for every $\nu \geq 0$

$$
\lim _{n \rightarrow \infty} \sigma_{p_{n}}^{D}(z)=f_{\nu}(z) \quad \text { compactly on } G_{\nu} .
$$

Proof. We define a holomorphic function $g: \bigcup_{\nu \geq 1} G_{\nu} \rightarrow \mathbf{C}$ by setting

$$
g(z)=f_{\nu}(z) \text { if } z \in G_{\nu}, \nu \geq 1 .
$$

For every fixed $\nu \geq 0$ and $n \geq 0$, let $K_{n, \nu}$ be the compact set consisting of all points in $G_{\nu}$ whose moduli do not exceed $(n+1)$ and whose distance to the complement of $G_{\nu}$ is at least $1 /(n+1)$. It is easy to see that $K_{n, \nu}$ has a connected complement and the sets

$$
K_{n}=K_{n, 0}, \quad L_{n}=\bigcup_{\nu=1}^{n} K_{n, \nu}
$$

do not separate the plane. Applying the theorem to the triples $\left(G_{0}, L_{n}, g\right)$ we obtain from (6) that, for every fixed $n \geq 0$, there are infinitely many indices $p \geq 0$ such that

$$
\left|\sigma_{p}^{D}(z)-f(z)\right|<\varepsilon_{n} \quad \text { for all } z \in K_{n}
$$

and

$$
\left|\sigma_{p}^{D}(z)-g(z)\right|<\varepsilon_{n} \quad \text { for all } z \in L_{n}
$$


where $\left(\varepsilon_{n}\right)$ is an arbitrary sequence of positive numbers tending to zero. We can rewrite the last two inequalities in the form

$$
\left|\sigma_{p}^{D}(z)-f_{\nu}(z)\right|<\varepsilon_{n} \text { for all } z \in K_{n, \nu} \text { where } 0 \leq \nu \leq n .
$$

Thus we can choose an index $p_{0}$ such that

$$
\left|\sigma_{p_{0}}^{D}(z)-f_{0}(z)\right|<\varepsilon_{0} \quad \text { for all } z \in K_{0,0}
$$

and then inductively determine indices $p_{n}$ such that $p_{n}>p_{n-1}$ for $n \geq 1$ and

$$
\left|\sigma_{p_{n}}^{D}(z)-f_{\nu}(z)\right|<\varepsilon_{n} \text { for all } z \in K_{n, \nu}, \text { where } 0 \leq \nu \leq n .
$$

Since, for each $\nu \geq 0$, every compact subset of $G_{\nu}$ is contained in $K_{n} \cup L_{n}$ for large $n$ the result follows from (9).

3. Proof of the theorem. The weights $\left(d_{k}\right)$ of the method $D$ will be constructed from the coeffients of a sequence of polynomials whose existence is guaranteed by the following result in [11].

THEOREM A. Let $G$ be a simply connected region which contains $\AA_{r}$ but no pole of $f$ (where $f, r$ are as defined in \$2). Suppose that $K$ is a compact subset of $G$ and that $L$ is a compact set which does not separate the plane and contains no point of $G \cup P \cup \Delta_{r}$. Then, for every $g \in A(L)$ and for every $\varepsilon>0$, there exists a polynomial $p(z)=\sum_{k=0}^{N} a_{k} z^{k}$ with the following properties:

$(\alpha)\left|a_{k}\right|<\varepsilon$ for $k=0,1, \ldots, N$,

$(\beta) p(1)=1$,

( $\gamma) \sum_{k=0}^{N}\left|a_{k}\right|<1+\varepsilon$,

( $\delta)\left|\sum_{k=0}^{N} a_{k}\left(\sum_{m=0}^{k} c_{m} z^{m}\right)-f(z)\right|<\varepsilon$ for all $z \in K$,

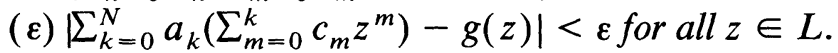

We need the following topological result as well as Theorem A to prove the theorem.

LEMMA. There exists a non-empty, countable collection $\mathscr{C}$ of pairs of compact sets $(K, L)$ with the following properties:

(a) For every $(K, L) \in \mathscr{C}, L$ does not separate the plane and contains no pole of $f$.

(b) For every $(K, L) \in \mathscr{C}$, there exists a simply connected region $H$ that does not contain a pole of $f$ and satisfies the conditions $K \cup \AA_{r} \subset H$ and $L \cap\left(H \cup \Delta_{r}\right)=\varnothing$. 

of $f$,

(c) Given (i) any simply connected region G containing $\AA_{r}$ but no pole

(ii) any compact set $\Lambda$ that does not separate the plane and contains no point of $G \cup \Delta_{r} \cup P$, and

(iii) any compact subset $\Psi$ of $G$, then there exists a pair $(K, L) \in \mathscr{C}$ such that $\Psi \subset K \subset G$ and $\Lambda \subset L$.

Proof. Let $\mathscr{K}$ be the collection of all sets of the form $S_{1} \cup S_{2} \cup \cdots \cup$ $S_{p}$ where $S_{1}, \ldots, S_{p}$ are compact squares with complex rational ${ }^{1}$ vertices and horizontal sides. Let $\mathscr{L}$ be the collection of all sets that do not separate the plane, contain no pole of $f$, and can be written in the form

$$
\left(S_{1} \cup \cdots \cup S_{m}\right) \backslash\left(B_{1} \cup \cdots \cup B_{n}\right)
$$

where $B_{1}, \ldots, B_{n}$ are open discs with complex rational centres and rational radii. Clearly the product $\mathscr{K} \times \mathscr{L}$ is countable. Hence we obtain an at most countable set by defining $\mathscr{C}$ to be the set of all pairs $(K, L)$ in $\mathscr{K} \times \mathscr{L}$ for which there exists a simply connected region $H$ that does not contain a pole of $f$ and for which the conditions $K \cup \AA_{r} \subset H$ and $L \cap\left(H \cup \Delta_{r}\right)=$ $\varnothing$ hold.

From the definitions of $\mathscr{L}$ and $\mathscr{C}$ it's clear that $\mathscr{C}$ satisfies (a) and (b). To prove that (c) is also satisfied, suppose that $G, \Lambda, \Psi$ are as in (i), (ii), (iii). It is easy to see that there is a $K \in \mathscr{K}$ satisfying $\Psi \subset K \subset G$ and the most intricate part of the proof is to find an $L$ such that $\Lambda \subset L$ and $(K, L) \in \mathscr{C}$.

We first construct a simply connected region $H$ such that $K \cup \triangle_{r} \subset$ $H \subset G$ and $\Lambda \cap \bar{H}=\varnothing$. It can be shown by the Riemann mapping theorem that there is a connected compact subset $F$ of $G$ that contains $\{0\} \cup K$. (In fact, if $\phi: \AA \rightarrow G$ is a conformal mapping then we can choose $F=\phi\left(\Delta_{\rho}\right)$ for some $\rho<1$.) From (ii), it follows that $\Lambda$ is a positive distance, $e$, from $F \cup \Delta_{r}$ and so the set $B=\{w+z: z \in \Lambda$, $|w| \leq e / 2\}$ does not meet $F \cup \Delta_{r}$. Since $G$ is a simply connected region, $\mathrm{C}_{\infty} \backslash G$ is connected and so $B \cup\left(\mathbf{C}_{\infty} \backslash G\right)$ is a connected closed set. Its complement is $G \backslash B$, an open set, the components of which have connected complements in $\mathbf{C}_{\infty}$ and hence are simply connected. Since $F \cup \AA_{r}$ is a connected subset of $G \backslash B$, we can pick $H$ to be that component of $G \backslash B$ which contains $F \cup \stackrel{\triangle}{\Delta}_{r}$ and so ensure that $K \cup \AA_{r} \subset H \subset G$ and $\Lambda \cap \bar{H}=\varnothing($ since $\Lambda \subset \stackrel{\circ}{B})$.

We now construct an $L \in \mathscr{L}$ such that $\Lambda \subset L$ and $L \cap\left(H \cup \Delta_{r}\right)=\varnothing$ which will show that $(K, L)$ belongs to $\mathscr{C}$ and thus prove that (c) holds.

\footnotetext{
${ }^{1}$ I.e., complex numbers whose real and imaginary parts are rational.
} 
We know that $\Lambda$ is a positive distance $\delta$ from $P \cup \bar{H}$. Consider a grid on $C$ made up of squares with horizontal and vertical sides such that the corners of the squares are complex rational numbers and the length of the sides is less than $\delta / \sqrt{2}$. We can pick four vertices of this grid to obtain a rectangle $R$ with horizontal and vertical sides containing $\Lambda$. Let $S_{1}, S_{2}, \ldots, S_{m}$ be all the compact squares of the grid that intersect $\Lambda$. Thus $\Lambda \subset S_{1} \cup S_{2} \cup \cdots \cup S_{m}$ and $\left(S_{1} \cup S_{2} \cup \cdots S_{m}\right) \cap(P \cup \bar{H})=\varnothing$ because of the size of the squares.

$\left(S_{1} \cup S_{2} \cup \cdots \cup S_{m}\right)^{c}$ may be disconnected, but it consists of the union of $R^{c}$ and finitely many line segments and interiors of squares and so has finitely many components. We pick one point from each of these open components and join it to a fixed arbitrary point of $R^{c}$ by a path which lies entirely in the complement of $\Lambda$. Denoting the union of these paths by $\Gamma$, we see that $\left(S_{1} \cup S_{2} \cup \cdots \cup S_{m}\right)^{c} \cup \Gamma$ is connected and does not intersect $\Lambda$. Since $\Gamma$ is compact, it is a positive distance from $\Lambda$, and so can be covered by finitely many open discs $B_{1}, B_{2}, \ldots, B_{n}$ that have complex rational centres, rational radii and do not intersect $\Lambda$. Thus, defining $L=\left(S_{1} \cup S_{2} \cup \cdots \cup S_{m}\right) \backslash\left(B_{1} \cup B_{2} \cup \cdots \cup B_{n}\right)$, we find that $L^{c}=\left(S_{1} \cup S_{2} \cup \cdots \cup S_{m}\right)^{c} \cup\left(B_{1} \cup B_{2} \cup \cdots \cup B_{n}\right)$ is connected and $L \cap(P \cup \bar{H})=\varnothing$. Hence we have that $L \in \mathscr{L}, \Lambda \subset L$ and $L \cap(H \cup$ $\left.\Delta_{r}\right)=\varnothing$, which shows that $(K, L) \in \mathscr{C}$ and completes the proof of the lemma.

We come now to the proof of the theorem.

Proof. Let $\mathscr{C}$ be a countable collection of pairs of compact sets which satisfies the conditions (a), (b), (c) of the lemma. Denote by $\mathscr{Q}$ the set of all polynomials with complex rational coefficients. Since $\mathscr{2}$ is countable, the set $\mathscr{Q} \times \mathscr{C}$ is also countable and so the elements of $\mathscr{Q} \times \mathscr{C}$ can be enumerated by an infinite sequence $\left(\Omega_{n}\right)_{n \geq 0}$, say, in such a way that every element of $\mathscr{Q} \times \mathscr{C}$ occurs infinitely often among the $\Omega_{n}$.

Let $\left(\varepsilon_{n}\right)_{n \geq 0}$ be a sequence of positive numbers, and define for every $n \geq 0$

$$
g_{n}(z)= \begin{cases}f(z) & \text { for } z \in K_{n}, \\ q_{n}(z) & \text { for } z \in L_{n} .\end{cases}
$$

Let $h: \mathbf{N}_{0} \rightarrow \mathbf{N}_{0}$ be a function (to be specified later). Since, for every $m \geq 0,\left(K_{m}, L_{m}\right) \in \mathscr{C}$, it follows from condition (a) and (b) of the lemma that $K_{m}$ and $L_{m}$ satisfy the topological hypotheses of Theorem A. Thus we obtain polynomials $p_{n}(z)=\sum_{k=0}^{\infty} a_{n, k} z^{k}$ where $a_{n, k}=0$ for $k>l_{n}$, say, 
such that the following conditions hold for every $n \geq 0$ :

$$
\begin{gathered}
\left|a_{n, k}\right|<\varepsilon_{n} \text { for } k=0,1, \ldots, \\
\sum_{k=0}^{l_{n}} a_{n, k}=1, \\
\sum_{k=0}^{l_{n}}\left|a_{n, k}\right|<1+\varepsilon_{n}, \\
\left|\sum_{k=0}^{l_{n}} a_{n, k}\left(\sum_{m=0}^{k} c_{m} z^{m}\right)-g_{h(n)}(z)\right|<\varepsilon_{n} \text { for all } z \in K_{h(n)} \cup L_{h(n)} .
\end{gathered}
$$

For reasons that will become apparent later we define the sequence $\left(\varepsilon_{n}\right)_{n \geq 0}$ and the function $h$ inductively as follows. Let $\varepsilon_{0}=1, h(0)=0$ and supposing that $\varepsilon_{0}, \varepsilon_{1}, \ldots, \varepsilon_{n}$ and $h(0), h(1), \ldots, h(n)$ have been defined set

$$
\varepsilon_{n+1}=2^{-n-1} M_{n}^{-1}\left(l_{n}+1\right)^{-1}
$$

where

$$
M_{n}=1+\max \left\{\sum_{m=0}^{l_{n}}\left|c_{m} z^{m}\right|: z \in\{0\} \cup \bigcup_{\nu=0}^{h(n)}\left(K_{\nu} \cup L_{\nu}\right)\right\}
$$

and

$$
h(n+1)= \begin{cases}h(n) & \text { if }\left|g_{h(n)}(z)\right|+\left|A_{n}(z)\right|>\sqrt{n} \\ & \text { for some } z \in K_{h(n)} \cup L_{h(n)} \\ h(n)+1 & \text { otherwise, }\end{cases}
$$

where, in the notation of (5), $A_{n}(z)=\sum_{\mu=0}^{n}\left(\sigma_{\mu}^{A}(z)-g_{h(n)}(z)\right)$.

It is important to note that $h: \mathbf{N}_{0} \rightarrow \mathbf{N}_{0}$ is a non-decreasing surjection. To see this, since $h(0)=0$ and $h(n+1) \in\{h(n), h(n)+1\}$, if $h$ is not a surjection there exists a non-negative integer $N$ such that $h(n)=h(N)$ for all $n \geq N$. Thus for every $n>N$ and every $z \in K_{h(n)} \cup L_{h(n)}$ we would obtain from (13) the inequality

$$
\begin{aligned}
\left|A_{n}(z)\right| & =\left|A_{N}(z)+\sum_{\mu=N+1}^{n}\left(\sigma_{\mu}^{A}(z)-g_{h(\mu)}(z)\right)\right| \\
& \leq\left|A_{N}(z)\right|+\sum_{\mu=N+1}^{n}\left|\sigma_{\mu}^{A}(z)-g_{h(\mu)}(z)\right|<\left|A_{N}(z)\right|+\sum_{\mu=N+1}^{n} \varepsilon_{\mu} .
\end{aligned}
$$

Since $\varepsilon_{\mu}<2^{-\mu}$ by (14), this would imply that $\left|g_{h(n)}(z)\right|+\left|A_{n}(z)\right|$ was bounded by $\left|g_{h(N)}(z)\right|+\left|A_{N}(z)\right|+2^{-N}$ on $K_{h(n)} \cup L_{h(n)}$ which would contradict (15). 
Also note that the sequence $\left(l_{n}\right)$ is strictly increasing because, by (10) and (14), we have for $n \geq 0$

$$
\sum_{k=0}^{l_{n}}\left|a_{n+1, k}\right|<\sum_{k=0}^{l_{n}}\left(l_{n}+1\right)^{-1}=1 .
$$

Thus $l_{n+1}$ must be greater than $l_{n}$ as otherwise (11) could not hold.

Since $\left|a_{n, k}\right| \leq 2^{-n}$ (from (10) and (14)), we obtain a well-defined sequence of weights $\left(d_{k}\right)$ by setting

$$
d_{k}=\sum_{\mu=0}^{\infty} a_{\mu, k} \quad \text { for } k=0,1, \ldots
$$

Now consider the numbers $D_{n}=d_{0}+d_{1}+\cdots+d_{n}$. We can assume, without loss of generality, that for $n=1$ the conditions (10) through (13) hold with $\varepsilon_{1}$ replaced by $\tilde{\varepsilon}_{1}=\varepsilon_{1} / 2$. Then there is a positive $\delta$ such that for every polynomial $\tilde{p}_{1}(z)=\sum_{k=0}^{l_{1}} \tilde{a}_{1, k} z^{k}$ satisfying $\tilde{p}_{1}(1)=1$ and $\left|a_{1, k}-\tilde{a}_{1, k}\right|<\delta$ for $k=0,1, \ldots, l_{1}$, the conditions (10) through (13) are satisfied with $n=1$ and $a_{1, k}$ replaced by $\tilde{a}_{1, k}$. Making the corresponding changes in the series

$$
d_{0}=\sum_{\mu=0}^{\infty} a_{\mu, 0}, \quad d_{1}=\sum_{\mu=0}^{\infty} a_{\mu, 1}, \ldots, \quad d_{l_{1}-1}=\sum_{\mu=0}^{\infty} a_{\mu, l_{1}-1}
$$

we can always ensure that $D_{0}, D_{1}, \ldots, D_{l_{1}-1}$ are non-zero. Hence, without loss of generality, we can assume that $p_{1}(z)=\tilde{p}_{1}(z)$ and $D_{n} \neq 0$ for $n<l_{1}$.

We now show that $D_{n} \neq 0$ for $n \geq l_{1}$. To this end, suppose for some $m \geq 1 l_{m} \leq n<l_{m+1}$ so that $D_{n}=\sum_{k=0}^{n} \sum_{\mu=0}^{\infty} a_{\mu, k}=\sum_{\mu=0}^{\infty} \sum_{k=0}^{n} a_{\mu, k}$. Now

$$
\sum_{\mu=m+2}^{\infty} \sum_{k=0}^{n}\left|a_{\mu, k}\right| \leq \sum_{\mu=k+2}^{\infty} \sum_{k=0}^{l_{m+1}}\left|a_{\mu, k}\right|<\sum_{\mu=m+2}^{\infty} \frac{\left(l_{m+1}+1\right) 2^{-\mu}}{M_{\mu-1}\left(l_{\mu-1}+1\right)}
$$

by (10) and (14) so that for $m=1,2, \ldots$

$$
\sum_{\mu=m+2}^{\infty} \sum_{k=0}^{n}\left|a_{\mu, k}\right|<\sum_{\mu=m+2}^{\infty} \frac{2^{-\mu}}{M_{\mu-1}}<1 .
$$

Hence

$$
\left|D_{n}-\sum_{\mu=0}^{m+1} \sum_{k=0}^{n} a_{\mu, k}\right|<\sum_{\mu=m+2}^{\infty} \frac{2^{-\mu}}{M_{\mu-1}}
$$

and since $a_{\mu, k}=0$ for $k>l_{\mu}$ and $\left(l_{n}\right)$ is increasing this can be written as

$$
\left|D_{n}-\sum_{\mu=0}^{m} \sum_{k=0}^{l_{\mu}} a_{\mu, k}-\sum_{k=0}^{n} a_{m+1, k}\right|<\sum_{\mu=m+2}^{\infty} \frac{2^{-\mu}}{M_{\mu-1}}
$$


which by (11) is

$$
\left|D_{n}-(m+1)-\sum_{k=0}^{n} a_{m+1, k}\right|<\sum_{\mu=m+2}^{\infty} \frac{2^{-\mu}}{M_{\mu-1}} .
$$

Using the triangle inequality and (12) gives

$$
\left|D_{n}-(m+1)\right|<\sum_{\mu=m+2}^{\infty} \frac{2^{-\mu}}{M_{\mu-1}}+\left(1+\varepsilon_{m+1}\right)
$$

and (14) gives

$$
\left|D_{n}-(m+1)\right|<1+\sum_{\mu=m+1}^{\infty} \frac{2^{-\mu}}{M_{\mu-1}}<2 .
$$

Thus $D_{n} \neq 0$ for $n \geq l_{1}$ and we have a well-defined weighted means method $D=\left(d_{n, k}\right)$ defined as in (1).

To show that $D$ is regular we first note that (2) follows directly from (18). To show that (3) holds, if $l_{m} \leq n<l_{m+1}$ then by (17)

$$
\sum_{k=0}^{n}\left|d_{k}\right| \leq \sum_{\mu=0}^{\infty} \sum_{k=0}^{n}\left|a_{\mu, k}\right|<\sum_{\mu=0}^{m+1} \sum_{k=0}^{l_{\mu}}\left|a_{\mu, k}\right|+1
$$

and by (12),

$$
\sum_{k=0}^{n}\left|d_{k}\right|<\sum_{\mu=0}^{m+1}\left(1+\varepsilon_{\mu}\right)+1 .
$$

Thus, by using (14) we get

$$
\left|D_{n}\right| \leq \sum_{k=0}^{n}\left|d_{k}\right|<\sum_{\mu=0}^{m+1}\left(1+2^{-\mu}\right)+1<m+5,
$$

and this together with (18) gives $\lim _{n \rightarrow \infty}\left|D_{n}\right|^{-1} \sum_{k=0}^{n}\left|d_{k}\right|=1$, which certainly implies (3). Hence, $D$ is regular.

Before attempting to prove the universal property of $D$ we first show that there is a subsequence $\left(\sigma_{j_{n}}^{D}\right)$ of $\left(\sigma_{n}^{D}\right)$ such that

$$
\lim _{\nu \rightarrow \infty} \max _{z \in K_{\nu} \cup L_{\nu}}\left|\boldsymbol{\sigma}_{j_{\nu}}^{D}(z)-g_{\nu}(z)\right|=0 .
$$

In fact, we choose $j_{\nu}=l_{n(\nu)}$ where $n(\nu)=\max \left\{n \in \mathbf{N}_{0} \mid h(n)=\nu\right\}$. It follows from (15) that $(n(\nu))_{\nu \geq 0}$ is a strictly increasing sequence and hence also $\left(j_{\nu}\right)_{\nu \geq 0}$ is strictly increasing. Moreover, it follows from (15) and the definition of $n(\nu)$ that $h(n(\nu))=\nu$ and for all $\nu \geq 0$

$$
\left|g_{\nu}(z)\right|+\left|A_{n(\nu)}(z)\right| \leq \sqrt{n(\nu)} \text { for all } z \in K_{\nu} \cup L_{\nu} .
$$


We can easily verify the identity

$$
\begin{aligned}
D_{j_{\nu}}\left(\sigma_{j_{\nu}}{ }(z)-g_{\nu}(z)\right)= & \left(n(\nu)+1-D_{j_{\nu}}\right) g_{\nu}(z)+A_{n(\nu)}(z) \\
& +\sum_{\mu=n(\nu)+1}^{\infty} \sum_{k=0}^{j_{\nu}} a_{\mu, k} \sum_{m=0}^{k} c_{m} z^{m} .
\end{aligned}
$$

By (18) and (20) we see that for all $\nu \geq 0$

$$
\left|\left(n(\nu)+1-D_{j_{\nu}}\right) g_{\nu}(z)+A_{n(\nu)}(z)\right| \leq 2 \sqrt{n(\nu)} \text { for all } z \in K_{\nu} \cup L_{\nu} .
$$

The modulus of the third term on the right-hand side of (21) does not exceed

$$
\begin{aligned}
& \sum_{\mu=n(\nu)+1}^{\infty} \sum_{k=0}^{j_{\nu}}\left|a_{\mu, k}\right| \sum_{m=0}^{j_{\nu}}\left|c_{m} z^{m}\right| \\
& \quad<\sum_{\mu=n(\nu)+1}^{\infty} \sum_{k=0}^{j_{\nu}} \frac{2^{-\mu}}{\left(l_{\mu-1}+1\right)} \cdot \frac{M_{n(\nu)}}{M_{\mu-1}} \quad \text { for all } z \in K_{\nu} \cup L_{\nu}
\end{aligned}
$$

by (10) and (14) (noting that $h(n(\nu))=\nu)$. Hence we get

$$
\left|\sum_{\mu=n(\nu)+1}^{\infty} \sum_{k=0}^{j_{\nu}} a_{\mu, k} \sum_{m=0}^{k} c_{m} z^{m}\right|<\sum_{\mu=n(\nu)+1}^{\infty} 2^{-\mu} \leq 1 \quad \text { for all } z \in K_{\nu} \cup L_{\nu} .
$$

Inserting these estimates into (21) we obtain for all $\nu \geq 0$

$$
\left|D_{j_{\nu}}\right|\left|\sigma_{j_{\nu}}^{D}(z)-g_{\nu}(z)\right| \leq 2 \sqrt{n(\nu)}+1 \quad \text { for all } z \in K_{\nu} \cup L_{\nu}
$$

so that

$$
\max _{z \in K_{\nu} \cup L_{\nu}}\left|\sigma_{j_{\nu}}^{D}(z)-g_{\nu}(z)\right| \leq \frac{(2 \sqrt{n(\nu)}+1)}{\left|D_{j_{\nu}}\right|} .
$$

Since $n(\nu)$ tends to infinity as $\nu$ tends to infinity and (18) holds, (19) follows easily.

To complete the proof of the theorem, suppose that $(G, L, g)$ is a triple satisfying (i), (ii), (iii). By Mergelyan's approximation theorem [8] there exists a sequence of polynomials $\left(\pi_{n}\right)_{n \geq 0}$ such that

$$
\lim _{n \rightarrow \infty} \max _{z \in L}\left|\pi_{n}(z)-g(z)\right|=0
$$

and we may assume, without loss of generality, that every $\pi_{n} \in \mathscr{Q}$ i.e. $\pi_{n}$ has complex rational coefficients. 
For every $n \geq 0$ let $\Psi_{n}$ be the compact set consisting of all points in $G$ whose moduli do not exceed $(n+1)$ and whose distance to the complement of $G$ is at least $1 /(n+1)$. The sequence $\left(\Psi_{n}\right)_{n \geq 0}$ exhausts $G$ i.e. for every compact set $K \subset G$ there is an index $n_{0}$ (depending on $K$ ) such that

$$
K \subset \Psi_{n} \text { for all } n \geq n_{0} .
$$

To prove (6) we now apply (c) of the lemma to the triple $\left(G, L, \Psi_{n}\right)$, for every $n \geq 0$, and hence obtain sequences $\left(\tilde{K}_{n}\right)_{n \geq 0},\left(\tilde{L}_{n}\right)_{n \geq 0}$ such that

$$
\left(\tilde{K}_{n}, \tilde{L}_{n}\right) \in \mathscr{C}, \quad \Psi_{n} \subset \tilde{K}_{n} \subset G \text { and } L \subset \tilde{L}_{n} \quad \text { for every } n \geq 0 .
$$

Since $\left(\pi_{0},\left(\tilde{K}_{0}, \tilde{L}_{0}\right)\right)$ belongs to $Q \times \mathscr{C}$ there is an index $\nu_{0}$ such that $q_{\nu_{0}}=\pi_{0}, K_{\nu_{0}}=\tilde{K}_{0}$ and $L_{\nu_{0}}=\tilde{L}_{0}$. Moreover, we can inductively choose indices $\nu_{n}(n \geq 1)$ such that $\left(\nu_{n}\right)_{n \geq 0}$ is strictly increasing and

$$
q_{\nu_{n}}=\pi_{n}, \quad K_{\nu_{n}}=\tilde{K}_{n}, \quad L_{\nu_{n}}=\tilde{L}_{n} \quad \text { for every } n \geq 0
$$

(since $\left(q_{n},\left(K_{n}, L_{n}\right)\right)_{n \geq 0}$ represents every element of $\mathscr{Q} \times \mathscr{C}$ infinitely often.) Substituting $\nu_{n}$ for $\nu$ in (19) and writing $k_{n}$ for $j_{\nu_{n}}$ we obtain

$$
\lim _{n \rightarrow \infty} \max _{z \in K_{\nu_{n}} \cup L_{\nu_{n}}}\left|\sigma_{k_{n}}^{D}(z)-g_{\nu_{n}}(z)\right|=0 .
$$

But for every $n \geq 0, \Psi_{n} \subset \tilde{K}_{n}=K_{\nu_{n}}, L \subset \tilde{L}_{n}=L_{\nu_{n}}$ and $\pi_{n}=q_{\nu_{n}}$ so that using the definition of $g_{v_{n}}$ we get

$$
\lim _{n \rightarrow \infty} \max _{z \in \Psi_{n}}\left|\boldsymbol{\sigma}_{k_{n}}^{D}(z)-f(z)\right|=0
$$

and

$$
\lim _{n \rightarrow \infty} \max _{z \in L}\left|\sigma_{k_{n}}^{D}(z)-\pi_{n}(z)\right|=0 .
$$

If $K$ is any compact subset of $G$ then (23) and (25) imply that

$$
\lim _{n \rightarrow \infty} \sigma_{k_{n}}^{D}(z)=f(z) \quad \text { uniformly on } K,
$$

and (22) with (26) gives

$$
\lim _{n \rightarrow \infty} \sigma_{k_{n}}^{D}(z)=g(z) \quad \text { uniformly on } L .
$$

Thus (6) holds and hence the result.

\section{REFERENCES}

[1] C. K. Chui and M. N. Parnes, Approximation by overconvergence of a power series, J. Math. Anal. Appl., 36 (1971), 693-696.

[2] K. Faulstich, Summierbarkeit von Potenzreihen durch Riesz-Verfahren mit komplexen Erzeugendenfolgen, Mitt. Math. Sem. Gießen, Heft 139 (1979). 
[3] K. Faulstich, W. Luh and L. Tomm, Universelle Approximation durch riesz-Transformierte der geometrischen Reihe, Manuscripta Math. 36 (1981), 309-321.

[4] G. H. Hardy, Divergent Series, (Oxford) 1949.

[5] W. Luh, Approximation analytischer Funktionen durch überkonvergente Potenzreihen und deren Matrix-Transformierten, Mitt. Math. Sem. Gie $\beta$ en, Heft 88 (1970).

[6] _ Über die Summierbarkeit der geometrischen Reihe, Mitt. Math. Sem. Gießen, Heft 113 (1974).

[7] _ Über den Satz von Mergelyan, J. Approximation Theory, 16 (1976), 194-198.

[8] S. N. Mergelyan, Uniform approximations of functions of a complex variable (Russian); Uspehi Mat. Nauk, 7 (1952), 31-122.

[9] A. Peyerimhoff, Lectures on Summability, Springer Lecture Notes in Mathematics No. 107, (1969).

[10] L. Tomm, Über die Summierbarkeit der geometrischen Riehe mit regulären Verfahren, Dissertation, Ulm (1979).

[11] _ A summability approximation theorem for Taylor series of meromorphic functions, J. Reine Angew. Math., 339 (1983), 133-146.

[12] L. Tomm and R. Trautner, A universal power series for approximation of measurable functions, Analysis, 2 (1982), 1-6.

Received February 25, 1983 and in revised form February 27, 1984. This paper was written at The University of Western Ontario, Canada where both authors were visiting research associates. We would like to thank the members of the Mathematics Department for their hospitality during our stay.

THE UNIVERSITY

BIRMINGHAM, B15 2TT, U.K.

AND

UNIVERSITÄT ULM

ABT. F. MATHEMATIK IV

7900 ULM (DONAU)

OBERER ESELSBERG, F.R.G. 



\section{PACIFIC JOURNAL OF MATHEMATICS EDITORS}

DONALD BABBITT (Managing Editor)
University of California
Los Angeles, CA 90024
CHARLES R. DEPrIMA
California Institute of Technology
Pasadena, CA 91125
R. FINN
Stanford University
Stanford, CA 94305

\author{
HERMANN FLASCHKa \\ University of Arizona \\ Tucson, AZ 85721 \\ RAMESH A. GANGOLLI \\ University of Washington \\ Seattle, WA 98195 \\ ROBION KIRBY \\ University of California \\ Berkeley, CA 94720 \\ C. C. Moore \\ University of California \\ Berkeley, CA 94720
}

Hugo Rossi

University of Utah

Salt Lake City, UT 84112

H. SAMELSON
Stanford University
Stanford, CA 94305

HAROLD STARK

University of California, San Diego

La Jolla, CA 92093

\section{ASSOCIATE EDITORS}
R. ARens
E. F. BECKENBACH (1906-1982)
B. H. NeUmanN
F. WOLF
K. YOSHIDA

\begin{tabular}{ll}
\multicolumn{2}{c}{ SUPPORTING INSTITUTIONS } \\
UNIVERSITY OF ARIZONA & UNIVERSITY OF OREGON \\
UNIVERSITY OF BRITISH COLUMBIA & UNIVERSITY OF SOUTHERN CALIFORNIA \\
CALIFORNIA INSTITUTE OF TECHNOLOGY & STANFORD UNIVERSITY \\
UNIVERSITY OF CALIFORNIA & UNIVERSITY OF HAWAII \\
MONTANA STATE UNIVERSITY & UNIVERSITY OF TOKYO \\
UNIVERSITY OF NEVADA, RENO & UNIVERSITY OF UTAH \\
NEW MEXICO STATE UNIVERSITY & WASHINGTON STATE UNIVERSITY \\
OREGON STATE UNIVERSITY & UNIVERSITY OF WASHINGTON
\end{tabular}

The Supporting Institutions listed above contribute to the cost of publication of this Journal, but they are not owners or publishers and have no responsibility for its content or policies.

\footnotetext{
Mathematical papers intended for publication in the Pacıfic Journal of Mathematıcs should be in typed form or offset-reproduced (not dittoed), double spaced with large margins. Please do not use built up fractions in the text of the manuscript. However, you may use them in the displayed equations. Underline Greek letters in red, German in green, and script in blue. The first paragraph must be capable of being used separately as a synopsis of the entire paper. In particular it should contain no bibliographic references. Please propose a heading for the odd numbered pages of less than 35 characters. Manuscripts, in triplicate, may be sent to any one of the editors. Please classify according to the scheme of Math. Rev ews, Index to Vol. 39. Supply name and address of author to whom proofs should be sent. All other communications should be addressed to the managing editor, or Elaine Barth, University of California, Los Angeles, California 90024.

There are page-charges associated with articles appearing in the Pacific Journal of Mathematics. These charges are expected to be paid by the author's University, Government Agency or Company. If the author or authors do not have access to such Institutional support these charges are waived. Single authors will receive 50 free reprints; joint authors will receive a total of 100 free reprints. Additional copies may be obtained at cost in multiples of 50 .
}

The Pacific Journal of Mathematics is issued monthly as of January 1966. Regular subscription rate: $\$ 190.00$ a year (5 Vols., 10 issues). Special rate: $\$ 66.00$ a year to individual members of supporting institutions.

Subscriptions, orders for numbers issued in the last three calendar years, and changes of address should be sent to Pacific Journal of Mathematics, P.O. Box 969, Carmel Valley, CA 93924, U.S.A. Old back numbers obtainable from Kraus Periodicals Co., Route 100, Millwood, NY 10546.

The Pacific Journal of Mathematics at P.O. Box 969, Carmel Valley, CA 93924 (ISSN 0030-8730) publishes 5 volumes per year. Application to mail at Second-class postage rates is pending at Carmel Valley, California, and additional mailing offices. Postmaster: Send address changes to Pacific Journal of Mathematics, P.O. Box 969, Carmel Valley, CA 93924.

PUBLISHED BY PACIFIC JOURNAL OF MATHEMATICS, A NON-PROFIT CORPORATION Copyright $\odot 1985$ by Pacific Journal of Mathematics 


\section{Pacific Journal of Mathematics}

\section{Vol. 117, No. 2 \\ February, 1985}

Robert Walter Bagley, Ta-Sun Wu and J. S. Yang, On a class of topological groups more general than SIN groups ............... 209

Bruce Alan Barnes, Algebraic elements of a Banach algebra modulo an ideal ...............................................219

Howard D. Fegan and Peter Gilkey, Invariants of the heat equation .......233

Erica Flapan, Necessary and sufficient conditions for certain homology

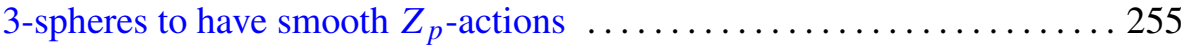

Philip R. Heath, Product formulae for Nielsen numbers of fibre maps . . . . 267

Derbiau Frank Hsu and A. Donald Keedwell, Generalized complete mappings, neofields, sequenceable groups and block designs. II ...... 291

Taqdir Husain, Orthogonal primitive idempotents and Banach algebras

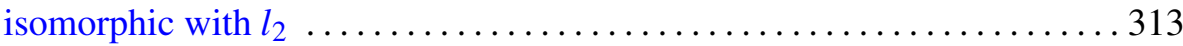

Gary M. Lieberman, Regularized distance and its applications . . . . . . . 329

William W. Menasco, Determining incompressibility of surfaces in alternating knot and link complements ............................ 353

Benjamin Muckenhoupt, Weighted reverse weak type inequalities for the Hardy-Littlewood maximal function $\ldots \ldots \ldots \ldots \ldots \ldots \ldots \ldots \ldots \ldots \ldots \ldots$

John Dacey O'Neill, Direct summands of direct products of slender

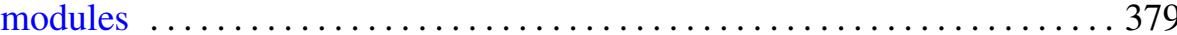

Andreas Rapp, Elimination of Malitz quantifiers in stable theories 387

Francisco José Ruiz, A unified approach to Carleson measures and $A_{p}$ weights

Hanamatagouda Pandappa Sankappanavar, Heyting algebras with dual

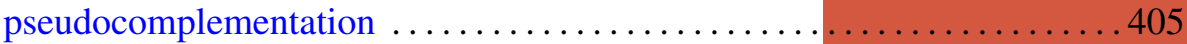

Annette Sinclair, Strong Carleman and strong uniform approximation . . . . 417

Masaaki Suzuki, The generalized Schwarz lemma for the Bergman metric

Brian Thorpe and Ludwig Tomm, Universal approximation by regular

weighted means 